\section{(6) OPEN ACCESS}

\title{
Developing a core outcome set for fistulising perianal Crohn's disease
}

\author{
Kapil Sahnan, ${ }^{1,2}$ Phil J Tozer, ${ }^{1,2}$ Samuel O Adegbola, ${ }^{1,2}$ Matthew J Lee, ${ }^{3}$ \\ Nick Heywood, ${ }^{4}$ Angus G K McNair, ${ }^{5}$ Daniel Hind, ${ }^{6}$ Nuha Yassin, ${ }^{1}$ Alan J Lobo, ${ }^{3}$ \\ Steven R Brown, ${ }^{3}$ Shaji Sebastian, ${ }^{7}$ Robin K S Phillips, ${ }^{1,2}$ Phillip F C Lung, ${ }^{1,2}$ \\ Omar D Faiz, ${ }^{1,2}$ Kay Crook, ${ }^{1}$ Sue Blackwell, ${ }^{8}$ Azmina Verjee, ${ }^{8}$ Ailsa L Hart, ${ }^{1,2}$ \\ Nicola S Fearnhead, ${ }^{9}$ the ENiGMA collaborators
}

\begin{abstract}
Additional material is published online only. To view please visit the journal online (http://dx.doi.org/10.1136/ gutjnl-2017-315503)
\end{abstract}

${ }^{1}$ Fistula Research Unit, St Mark's Hospital and Academic Institute, London, UK

${ }^{2}$ Department of Surgery and Cancer, Imperial College, St Mary's Hospital, London, UK ${ }^{3}$ Department of Colorectal Surgery, Sheffield Teaching Hospitals NHS Foundation Trust, Sheffield, UK

${ }^{4}$ Department of Colorectal

Surgery, University Hospitals of South Manchester, Manchester UK

${ }^{5}$ School of Social and

Community Medicine, University of Bristol, Bristol, UK

${ }^{6}$ Clinical Trials Research Unit, School of Health and Related Research, Sheffield, UK ${ }^{7}$ Department of

Gastroenterology, Hull and East Yorkshire NHS trust, Hull, UK

${ }^{8}$ Patient Representative, London, UK

${ }^{9}$ Department of Colorectal Surgery, Cambridge University Hospitals, Cambridge, UK

\section{Correspondence to}

Kapil Sahnan, Department of Surgery and Cancer, Imperia College, St Mary's Hospital, LondonW21NY, UK; ks303@ doctors.org.uk

Received 19 October 2017 Revised 15 December 2017 Accepted 18 December 2017 Published Online First

3 February 2018

\begin{abstract}
Objective Lack of standardised outcomes hampers effective analysis and comparison of data when comparing treatments in fistulising perianal Crohn's disease ( $p C D)$. Development of a standardised set of
\end{abstract} outcomes would resolve these issues. This study provides the definitive core outcome set (COS) for fistulising pCD. Design Candidate outcomes were generated through a systematic review and patient interviews. Consensus was established via a three-round Delphi process using a 9-point Likert scale based on how important they felt it was in determining treatment success culminating in a final consensus meeting. Stakeholders were recruited nationally and grouped into three panels (surgeons and radiologists, gastroenterologists and IBD specialist nurses, and patients). Participants received feedback from their panel (in the second round) and all participants (in the third round) to allow refinement of their scores.

Results A total of 295 outcomes were identified from systematic reviews and interviews that were categorised into 92 domains. 187 stakeholders (response rate $78.5 \%$ ) prioritised 49 outcomes through a three-round Delphi study. The final consensus meeting of 41 experts and patients generated agreement on an eight domain COS. The COS comprised three patient-reported outcome domains (quality of life, incontinence and a combined score of patient priorities) and five clinician-reported outcome domains (perianal disease activity, development of new perianal abscess/sepsis, new/recurrent fistula, unplanned surgery and faecal diversion).

Conclusion A fistulising pCD COS has been produced by all key stakeholders. Application of the COS will reduce heterogeneity in outcome reporting, thereby facilitating more meaningful comparisons between treatments, data synthesis and ultimately benefit patient care.

\section{INTRODUCTION}

The management of fistulising perianal Crohn's disease $(\mathrm{pCD})$ remains challenging. Fistulas are often complex in nature, and recurrence after treatment is common. Perianal manifestations of Crohn's disease are recognised in the Montreal classification as a distinct phenotype ${ }^{1}$ from luminal disease. Fistulising pCD is associated with significant morbidity and reduced quality of life. ${ }^{2}$ The

\section{Significance of this study}

What is already known on this subject?

- There is heterogeneity in the outcome measures used in interventional studies in fistulising perianal Crohn's disease. This limits metaanalysis and other methods for comparing treatment options in this disease.

What are the new findings?

- The generation of a patient-centred core outcome set (COS) based on the principles set out by the Core Outcome Measures in Effectiveness Trials (COMET) initiative and using a Delphi consensus of stakeholders including patients and clinicians who regularly manage fistulising perianal Crohn's disease.

How might it impact on clinical practice in the foreseeable future?

- This COS will form the basis of outcome measurement in future interventional studies of fistulising perianal Crohn's disease.

established treatment pathway is multidisciplinary and involves management of proctitis and drainage of sepsis, prior to optimisation of medical treatment, usually with a combination of thiopurine and antitumour necrosis factor (TNF) therapies. ${ }^{4}$ Initial drainage of sepsis and placement of loose setons are the mainstay of surgical treatment, although reparative surgery aimed at fistula closure may be offered in selected patients, and defunctioning stoma or proctectomy may sometimes be required. Undrained ongoing perianal sepsis, injudicious surgery and recurrent perianal sepsis may all result in a poor functional outcome for the patient.

There has been significant recent innovation in managing fistulising pCD both in biological therapy with trials studying fistula healing as a primary outcome measure ${ }^{5}$ and in the introduction of novel, sphincter preserving techniques ${ }^{6-8}$ as well as modification of existing operations, such as the BioLIFT $^{9}$ or LIFT-Plug. ${ }^{10}$ There are restrictions in the current literature that impair evidence synthesis and meta-analysis. One limitation is a lack of standardised outcome measurement, which hampers 
effective analysis and comparison of techniques and leads to a high risk of reporting bias. ${ }^{11}$ Most importantly, the currently used outcomes lack relevance to patients.

Measuring success or failure should not be determined by researchers alone, and the views of patients and other healthcare professionals involved in the care of patients with perianal Crohn's fistula must be considered. The Core Outcome Measures in Effectiveness Trials (COMET; www.comet-initiative.org/initiative) initiative has improved understanding of outcome reporting and standardised outcome reporting through the development of core outcome sets (COS). COSs are the minimum outcomes that should be reported in every study of a given condition. ${ }^{12}$ They have usually been informed by a systematic review and developed through a Delphi consensus process with key stakeholders.

COS are not restrictive; triallists may choose to investigate other outcomes but should always include the COS as a minimum within their study design. New COS are increasingly being developed, ${ }^{13}$ and they are widely recognised in a number of specialties, such as rheumatology, ${ }^{14}$ paediatric surgery ${ }^{15}$ and colorectal surgery. ${ }^{16}$ Increasingly, funding bodies advocate the inclusion of COS within proposed trial methodology ${ }^{16}$ and uptake among triallists is increasing. ${ }^{17}$

The aim of this study was to develop a COS for fistulising pCD using Delphi methodology.

\section{METHODS}

\section{Protocol registry}

The development of this COS is based on the principles advised by the COMET initiative ${ }^{18}$ and reported in accordance with the Core Outcome Set-STAndards for Reporting (COS-STAR) Statement. ${ }^{19}$ This study has been registered with the COMET initiative (www.comet-initiative.org).

\section{Scope}

The scope of the COS is to include all medical treatments and surgical interventions used alone or in combination for adult patients with fistulising pCD. Most patients with Crohn's fistula will undergo both medical and surgical interventions and studies usually follow a combined multidisciplinary stepwise approach, even when this is not explicit. The multimodal approach is used irrespective of whether the treatment intention is to induce fistula healing or palliate symptoms. A COS describes what outcomes should be measured but does not stipulate how they should be measured. It can be used for all types of study design, including audit.

\section{Overview}

In phase 1 , a long list of candidate outcomes that could be measured in fistulising pCD trials was identified, and outcomes were categorised into domains.

In phase 2, outcome domains were presented via a web-based Delphi system that was used to assess key stakeholders' views on the importance of each domain.

In phase 3, a consensus meeting with all stakeholders was used to finalise and ratify the COS.

\section{Participants}

Stakeholder representation was chosen to correlate with the clinical scenario since patients with perianal Crohn's fistulas are managed by multidisciplinary teams, including surgeons, gastroenterologists, radiologists and IBD specialist nurses. Inclusion
Box 1 Stakeholder groups demonstrating the three panels

\section{Panel SuRa}

- Colorectal surgeons (Su)

- Association of Coloproctology of Great Britain and Ireland with a declared specialist interest in managing IBD.

- Radiologists (Ra)

- British Society of Gastrointestinal and Abdominal Radiology.

\section{Panel GaNu}

- Gastroenterologists (Ga)

- British Society of Gastroenterology IBD section.

- IBD specialist nurses $(\mathrm{Nu})$

- Royal College of Nursing regional and national network group of IBD nurses.

\section{Panel Pa}

- Patients (Pa)

- Crohn's and Colitis United Kingdom, for Crohns, 'St Mark's Patient panel', and lleostomy Association (IA) (The ileostomy and internal pouch Support Group).

was limited to holding a consultant position or being on the IBD specialist nurse register.

All stakeholders were recruited through national organisations (and their subcommittees), and the study management group agreed that this should be limited to the UK to facilitate the process of ensuring equal though broad representation. The stakeholders were divided into three panels. Patients were given a panel to themselves as they were considered essential stakeholders (box 1).

A participant information sheet was available on the webpage of the organisations and charities. On registration, participants were again provided with information about the survey and invited to complete an initial registration survey to capture demographic data. One reminder was sent if no response was received after 2 weeks. A purposive sampling technique was used to ensure variation based on geographical regions.

There are limited data to inform severity stratification or prognostic classification of patients with Crohn's fistula, an 'unmet need' recognised internationally. ${ }^{20}$ After discussion within the study management group, it was decided that 'health states' would be a more useful way of categorising patients.

Four groups were determined by the study management group to reflect the various health states patients with fistulising $\mathrm{pCD}$ patients may be in.

1. 'This is my first anal fistula causing me symptoms'.

2. 'I've had at least one anal fistula before which got better but now I've got a new or newly symptomatic anal fistula'.

3. 'I have had anal fistula in the past and currently. They never completely settle and always give me symptoms'.

4. 'My fistula has healed following intervention'.

We aimed to invite 180 experts to ensure 70 experts with a $40 \%$ response rate. To maximise ongoing commitment to the process, we offered acknowledgement of participants completing all three rounds on publication of the study and sent newsletters after each of the Delphi rounds.

\section{Information sources}

A list of candidate outcomes were generated from a systematic literature review, interviews with patients, a dedicated patient 
Table 1 Demographics of stakeholders

\begin{tabular}{|c|c|c|c|c|c|c|}
\hline \multirow[b]{2}{*}{ Participants characteristics } & \multicolumn{3}{|c|}{ Registered participants $(n=238)$} & \multicolumn{3}{|c|}{ Consensus meeting $(n=47)$} \\
\hline & Clinicians & Patients & Total & Clinicians & Patients & Total \\
\hline \multicolumn{7}{|l|}{ Gender } \\
\hline Male & 108 & 18 & 126 & 23 & 2 & 25 \\
\hline Female & 45 & 65 & 110 & 10 & 12 & 22 \\
\hline Did not answer & 2 & 0 & 2 & 0 & 0 & 0 \\
\hline \multicolumn{7}{|l|}{ Region } \\
\hline Northern England & 37 & 18 & 55 & 8 & 2 & 10 \\
\hline Midland & 22 & 8 & 30 & 5 & 1 & 6 \\
\hline Southeast England & 58 & 33 & 91 & 13 & 9 & 22 \\
\hline Southwest England & 20 & 8 & 28 & 3 & 2 & 5 \\
\hline Wales & 6 & 6 & 12 & 1 & 0 & 1 \\
\hline Scotland & 7 & 6 & 13 & 1 & 0 & 1 \\
\hline Northern Ireland & 2 & 3 & 5 & 2 & 0 & 2 \\
\hline Did not answer & 3 & 1 & 4 & 0 & 0 & 0 \\
\hline \multicolumn{7}{|l|}{ Type of hospital } \\
\hline DGH & 56 & 64 & 120 & 10 & 7 & 17 \\
\hline Tertiary unit & 97 & 17 & 114 & 23 & 7 & 30 \\
\hline Private & & 1 & 1 & 0 & 0 & 0 \\
\hline Other & & 1 & 1 & 0 & 0 & 0 \\
\hline Did not answer & 2 & 0 & 0 & 0 & 0 & 0 \\
\hline \multicolumn{7}{|l|}{ Other clinicians characteristics } \\
\hline \multicolumn{7}{|l|}{ Length of consultant appointment (years) } \\
\hline $0-5$ & 46 & & & 8 & & \\
\hline $6-10$ & 34 & & & 4 & & \\
\hline $11-20$ & 53 & & & 16 & & \\
\hline$>20$ & 20 & & & 5 & & \\
\hline \multicolumn{7}{|l|}{ Other patient characteristics } \\
\hline \multicolumn{7}{|l|}{ Age (years) } \\
\hline $20-29$ & & 32 & & & 3 & \\
\hline $30-39$ & & 27 & & & 4 & \\
\hline $40-49$ & & 17 & & & 5 & \\
\hline $50-59$ & & 5 & & & 2 & \\
\hline \multicolumn{7}{|l|}{ Years with Crohn's disease (years) } \\
\hline $0-5$ & & 25 & & & 4 & \\
\hline $6-10$ & & 19 & & & 2 & \\
\hline $11-20$ & & 24 & & & 6 & \\
\hline$>20$ & & 15 & & & 2 & \\
\hline \multicolumn{7}{|l|}{ Years with anal fistula (years) } \\
\hline $0-5$ & & 52 & & & 2 & \\
\hline $6-10$ & & 18 & & & 8 & \\
\hline $11-20$ & & 11 & & & 2 & \\
\hline$>20$ & & 1 & & & 0 & \\
\hline Did not answer & & & & & 2 & \\
\hline \multicolumn{7}{|l|}{ Fistula status } \\
\hline First anal fistula & & 24 & & & 2 & \\
\hline Previously healed anal fistula, now new fistula & & 12 & & & 2 & \\
\hline Recurrent anal fistula & & 32 & & & 4 & \\
\hline Fistula healed following intervention & & 12 & & & 1 & \\
\hline Did not answer & & 0 & & & 5 & \\
\hline
\end{tabular}

and public involvement (PPI) meeting ${ }^{21}$ and the study management group.

A systematic review of studies assessing medical, surgical and combined (medical/surgical) treatment of fistulising pCD was performed in accordance with a prospectively registered protocol (PROSPERO CRD42016039019).
The OVID SP version of MEDLINE (1950-2016) and Embase (1980-2016) were searched using validated terms for 'Crohn's disease', 'anorectal fistula' and 'randomized controlled trials or prospective studies' separated by the Boolean operator 'AND' (online supplementary file 1). This was supplemented with a free-text search of the same databases, using relevant keywords/ 
terms (including synonyms and variants), also separated by the Boolean operator 'AND'. The search was limited to studies conducted in human adults aged $\geq 18$ years old and to papers published between 1 January 2010 and 12 July 2016, in order to ensure that identified outcomes were contemporary and currently applicable.

Prospective studies (including cohort comparisons, case controls and case series) that reported on outcomes on an intervention (medical, surgical and combination) for patients with fistulising pCD and recruited $\geq 10$ patients were included. Systematic reviews were included, and the individual studies reviewed were searched to ensure complete capture. Evaluation of luminal studies not primarily targeted at perianal fistulas were included where a subgroup analysis was presented for patients with fistula. Excluded were studies where the fistulas were of a non-Crohn's aetiology, were not perianal or were not published in the English language.

Five independent reviewers (KS, SOA, PJT, MJL and NH) used predefined selection criteria to screen the studies, using Covidence Systematic Review Software (Veritas Health Innovation, Melbourne, Australia; available at www.covidence.org). Each study was reviewed by at least two independent reviewers. Studies were initially screened in abstract format before full-text review. Conflicts were resolved by discussion with recourse to senior investigators (AH and NSF) where necessary.

Reported outcomes were extracted verbatim and listed in preparation for categorisation into domains.

\section{Domain categorisation}

Three members of the study team, two researchers (KS and PJT) and a patient representative (AV), categorised similar outcomes identified from the systematic review into domains by consensus. Four members of the study team (KS, PJT, SOA and AV) assessed and categorised the transcripts from the patient interviews and from the PPI meeting to supplement the list generated from the systematic review. All the included outcomes were categorised into themes and presented as such throughout the rest of the process.

Discrepancies were resolved through discussion with the senior authors (NF and $\mathrm{AH}$ ). Overlapping domains between data sources were condensed, producing a final list of candidate outcomes. The study management group, consisting of all stakeholder groups, methodologists and patient representatives, then reviewed and finalised the domains. Outcomes that were felt to be solely applicable to luminal disease were excluded if all members of the study management group were in agreement.

The patient representatives (SB, AV) created plain English definitions for all outcomes under consideration. The long list of all possible outcomes was presented to the stakeholders through a web-based system purposefully designed to conduct a threephase Delphi process.

\section{Consensus process}

A three-round online Delphi process was used to prioritise outcomes. In each round, participants scored outcomes using the numerical scale suggested by the Grading of Recommendations Assessment, Development and Evaluation (GRADE) group (http://www.gradeworkinggroup.org). Scale 1-3 signified an outcome of limited importance (categorised as 'not important'), 4-6 signified important but not critical (categorised as 'fairly important') and 7-9 signified an outcome of critical importance (categorised as 'really important'). In addition, a free-text space was provided for stakeholders to comment on the outcome definitions.

In round 1, participants were asked to score each outcome based on how important they felt it was in deciding whether the overall treatment of [their/their patient's] $p C D$ was successful and to suggest additional outcomes they felt were important but which had not previously been scored. All newly suggested outcomes were reviewed by the study management group and taken forward for assessment in the second round if within the scope of the COS.

In round 2, participants were asked to score the outcomes again having been shown numerical and graphical representations demonstrating how others in their panel scored each outcome in the first round. They were also shown their own response from the first round. In round 3, participants rescored the outcomes having been shown numerical and graphical representations of how all panels scored outcomes in the previous round. They were also shown their response from the second round.

Each round was open for 4 weeks. A reminder email was sent to participants who had not completed the round after 2 weeks and then again at 3 weeks. The final reminder asked if participants were experiencing difficulties in completing the questionnaire or if they had decided not to participate further in the study. Participants who completed round 1 were invited to complete both round 2 and round 3. A newsletter was sent to all participants in between all rounds to update them on progress and modifications.

Outcomes that were prioritised during the Delphi process were discussed and voted on at a face-to-face consensus meeting. Electronic voting was used to maintain anonymity (ResponseCard, Turning Technologies, Belfast, UK). An initial vote, 'In,' 'Out' or 'unsure,' was followed by debate among participants, refinement of the wording of the consensus statement and then a second vote of 'in', 'out' or 'unsure'.

\section{Outcome scoring and consensus definition}

Outcomes were carried forward between rounds if more than $>70 \%$ of all participants scored them as 'really important' (7-9). Each outcome was assessed for heterogeneity between the panels using a histogram depicting median scores. In addition,

\begin{tabular}{|c|c|c|c|c|c|}
\hline \multicolumn{6}{|c|}{ Voting demographics } \\
\hline Panel & & Round 1 & Round 2 & Round 3 & Consensus meeting ( $\mathrm{n}$ ) \\
\hline \multirow[t]{2}{*}{ SuRa (n/N (\%)) } & Surgeons & $39 / 47(83)$ & $39 / 39(100)$ & $39 / 39(100)$ & 12 \\
\hline & Radiologists & $21 / 27(78)$ & $21 / 21(100)$ & $21 / 21(100)$ & 4 \\
\hline \multirow[t]{2}{*}{ GaNu (n/N (\%)) } & Gastroenterologists & $44 / 59(75)$ & $44 / 45(98)$ & $44 / 44(100)$ & 12 \\
\hline & Nurses & $17 / 22(77)$ & 15/17 (88) & $15 / 15(100)$ & 5 \\
\hline $\mathrm{Pa}(\mathrm{n} / \mathrm{N}(\%))$ & Patients & $66 / 83(80)$ & $57 / 66(86)$ & $59 / 64(92)$ & 14 \\
\hline
\end{tabular}




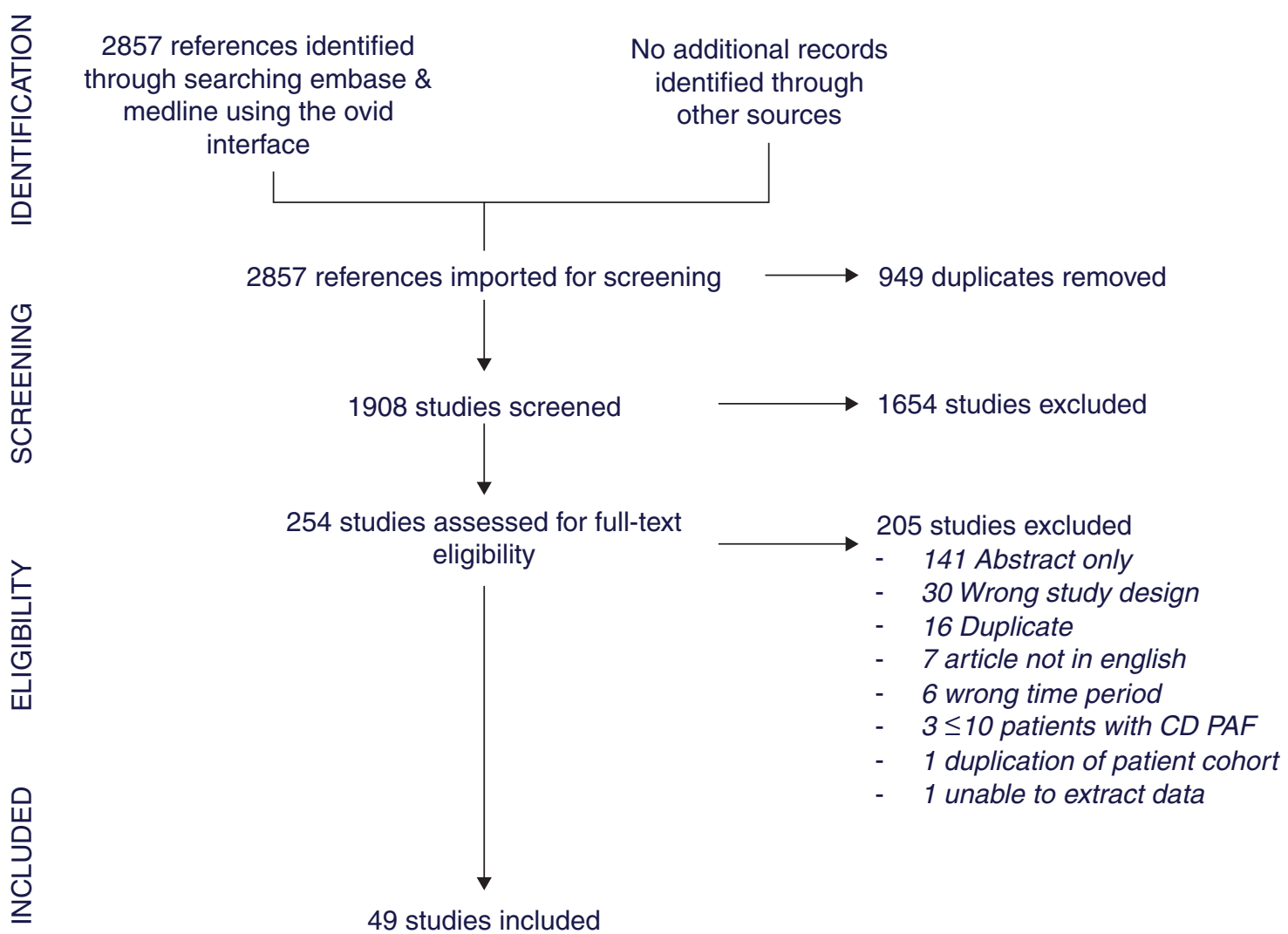

Figure 1 Preferred Reporting Items for Systematic Reviews and Meta-Analyses diagram of studies considered for the systematic review. CD, Crohn's disease; PAF, perianal fistula.

during each round, participants were given the opportunity to comment on the clarity and appropriateness of each outcome. All comments were reviewed by the study management group, and outcomes were modified to improve clarity if necessary.

At the end of each round, the study management group discussed all outcomes below the threshold for inclusion. The discussion was based on heterogeneity and any comments that had been recorded by the participants. Modification and frequently asked comments were sent to all participants using a newsletter in between rounds.

At the consensus meeting, participants were presented with the round 3 results and asked to vote prediscussion on whether they believed an outcome should be 'included in the COS', 'not included in the COS' or whether they were unsure. Participants were then asked to advocate either for inclusion or exclusion of an outcome, before repeating the voting. Those where $70 \%$ of participants voted for their inclusion were retained in the COS.

\section{Ethical considerations}

The local research and development department deemed the project to be service evaluation and therefore review by a National Health Service Research Ethics Committee was not necessary. The stakeholders were provided with information prior to registering to participate and throughout the process via newsletters. Consent to participate in the study was implied through completion of demographic questionnaires and voluntary attendance at the final meeting. Stakeholders were able to withdraw from the study at any time either by contacting the study team or by simply not responding to any of the questionnaires.

\section{RESULTS}

\section{Protocol modifications}

Following the analysis of the round 2 of the Delphi process, it was identified that that panel colorectal surgeons and radiologists

Table 3 Number of outcome measures reported and completeness of outcome reporting in perianal Crohn's fistula studies

\begin{tabular}{|c|c|c|}
\hline & $\begin{array}{l}\text { Randomised } \\
\text { studies }(n=10)\end{array}$ & $\begin{array}{l}\text { Non-randomised } \\
\text { studies }(n=39)\end{array}$ \\
\hline \multicolumn{3}{|l|}{ Year } \\
\hline 2010-2011 & 2 & 15 \\
\hline 2012-2013 & 2 & 11 \\
\hline 2014-2016 & 6 & 13 \\
\hline \multicolumn{3}{|l|}{ Intervention } \\
\hline Medical & 5 & 18 \\
\hline Surgical & 4 & 14 \\
\hline Combination & 1 & 7 \\
\hline \multicolumn{3}{|l|}{ Number of patients with fistula } \\
\hline$<50$ & 3 & 22 \\
\hline $50-100$ & 5 & 4 \\
\hline$>100$ & 1 & 3 \\
\hline NR & 1 & 9 \\
\hline \multicolumn{3}{|l|}{ Number of outcomes measures } \\
\hline$<5$ & 3 & 18 \\
\hline $5-10$ & 6 & 20 \\
\hline$>10$ & 1 & 1 \\
\hline $\begin{array}{l}\text { Meets all core criteria for completeness of } \\
\text { outcome reporting (\%) }\end{array}$ & 50.0 & 12.80 \\
\hline
\end{tabular}

Sahnan K, et al. Gut 2019;68:226-238. doi:10.1136/gutjnl-2017-315503 


\begin{tabular}{ll}
\hline Table 4 & The most commonly outcome measures reported \\
\hline Outcome measures in the included studies & $\begin{array}{l}\text { Number of } \\
\text { studies }\end{array}$ \\
\hline$\geq 50 \%$ tracts not draining on clinical examination & 22 \\
Perianal Disease Activity Index & 20 \\
Crohn's Disease Activity Index & 19 \\
Closure of external opening & 17 \\
\hline No drainage either spontaneously or on gentle finger pressure & 12 \\
\hline
\end{tabular}

(SuRa) were the most discriminatory and the panel patient was the least discriminatory. Using the prespecified criteria, only $6 / 79(7.6 \%)$ outcomes were below the threshold for exclusion for the patient panel compared with $45 / 79$ (57.0\%) from the most discriminatory panel ( $\mathrm{SuRa}$ ) (online supplementary file 2).

The criteria for retention in the second round of the Delphi process were therefore modified, so that not only were outcomes where $>70 \%$ participants overall scored them 7-9 retained but also those which $>90 \%$ of the patient panel had scored them 7-9 were carried forward to phase 3 . In addition, four outcomes (online supplementary file 2) were retained by the study management group as they were felt to be of key importance and would benefit from further evaluation and discussion.

High patient scoring and a change to the protocol in round 2 meant the study management group decided that no outcomes were to be excluded in round 3. Analysis of round 3 confirmed the decision as again the $70 \%$ cut-off was deemed insufficiently discriminatory (online supplementary file 3 ). This allowed for the participants in the consensus meeting to be given the opportunity to see all the remaining outcomes.

\section{Participants}

A total of 238 participants registered their interest. Of these, $187 / 238$ participants $(78.5 \%)$ registered their demographics and completed round 1 of the Delphi survey. One hundred and seventy-six out of 187 participants (94.1\%) completed round 2 and $183 / 187$ participants (97.9\%) completed round 3. In total, 47 participants attended the face-to-face meeting across the panels (16 from panel SuRa, 17 from panel gastroenterologists and IBD specialist nurses ( $\mathrm{GaNu}$ ) and 14 from panel patients (Pa)). Demographic details for each stakeholder group are summarised in tables 1 and 2 .

\section{Information sources and domain categorisation}

A total of 2857 titles were identified, of which 949 were duplicates and the remaining 1908 were screened. Of these, a further 1654 studies were excluded based on title and abstract review. Following full-text review, a further 205 papers were excluded for the following reasons: only the abstract could be found, non-prospective study design, non-English language publications or not having a sufficient number of patients with Crohn's fistula receiving treatment. This resulted in 49 included studies (figure 1). No additional papers were identified from systematic reviews or other trials. In total, 18 of the studies (37\%) were prospective cohort studies (including two studies where data were retrospectively analysed from a prospectively collected database), 18 (37\%) were systematic reviews and meta-analyses, $9(18 \%)$ were randomised controlled trials and the remainder were non-randomised studies. The median number of study participants with Crohn's perianal fistula who received treatment was 29 (IQR 17-68).

There were 295 different clinical outcomes reported, with studies reporting a median of six (IQR 3-7) outcomes; these are summarised in table 3 . The three most commonly reported outcome measures in the studies were: $\geq 50 \%$ of tracts not draining on clinical examination (22 studies; 45\%), Perianal Disease Activity Index (20 studies; 41\%) and Crohn's Disease Activity Index (CDAI) (table 4). No single outcome was reported in every study. The individual studies and the quality of outcome reporting were assessed using the five core questions proposed by Harman's criteria ${ }^{22}$ in online supplementary file 4 . Duplicate and analogous terms were merged to form 89 unique outcome domains. Eleven further outcomes were generated through a combination of patient interviews and the PPI day. The study management group added a further six. The resultant 106 outcomes were reviewed by the study management group, and 14 were excluded as they were felt to be applicable only to luminal disease, resulting in 92 unique outcomes that entered round 1 of the Delphi process. A summary of outcomes used in the online Delphi process with their lay definitions, organised according to themes, is presented in table 5 .

\section{Outcome prioritisation}

The 92 outcomes were reviewed by participants in round 1, which generated 201 individual comments. The study management group retained nine outcomes having altered the name (or lay description), due to polarising views/heterogeneity or comments by participants, implying a lack of clarity (online supplementary file 5). Five de novo outcomes were added, and 18 outcomes were excluded according to the preset criteria. At the end of round 1, interim analysis demonstrated that the top 10 outcomes rated by the panels were similar and are seen in table 6.

Of the 79 outcomes that entered round 2, 41 were below the preset threshold for exclusion. After a modification to the protocol, 12 of these were retained (eight due to high patient scoring and four by the study management group, as described above), which resulted in 29 (36.7\%) being dropped from round 2 (online supplementary file 2). The resultant 49 outcomes entered into the third round, and all were taken forward to the face-to-face consensus meeting (table 7). The process is described in figure 2, and individual scores for each round are in online supplementary files $2-4$.

\section{Consensus meeting}

Following scoring at the consensus meeting, eight outcomes were retained in the COS. This constituted three patient-reported and five clinician-reported outcome domains (box 2).

The outcome radiological outcome was felt to be important by the attendees at the consensus meeting but did not meet criteria for retention in the COS after consensus discussion. Attendees' main reason for excluding the outcome was that the cost associated with MRI was likely to prohibit its use in research across both low-income and high-income countries and that it was therefore not appropriate for inclusion in a COS.

The meeting attendees did however note that it should be recommended for use as an outcome in all studies where it was feasible to do so.

At the consensus meeting, the individual outcomes in the 'impact on life' theme did not meet universal consensus but were scored very highly by the patients and advocated for by them. Members of other panels felt the inclusion was important but were unable to differentiate the importance between them. As such, after a unanimous decision, it was decided that an individual outcome making up this overarching domain should be voted on and prioritised by patients alone. 


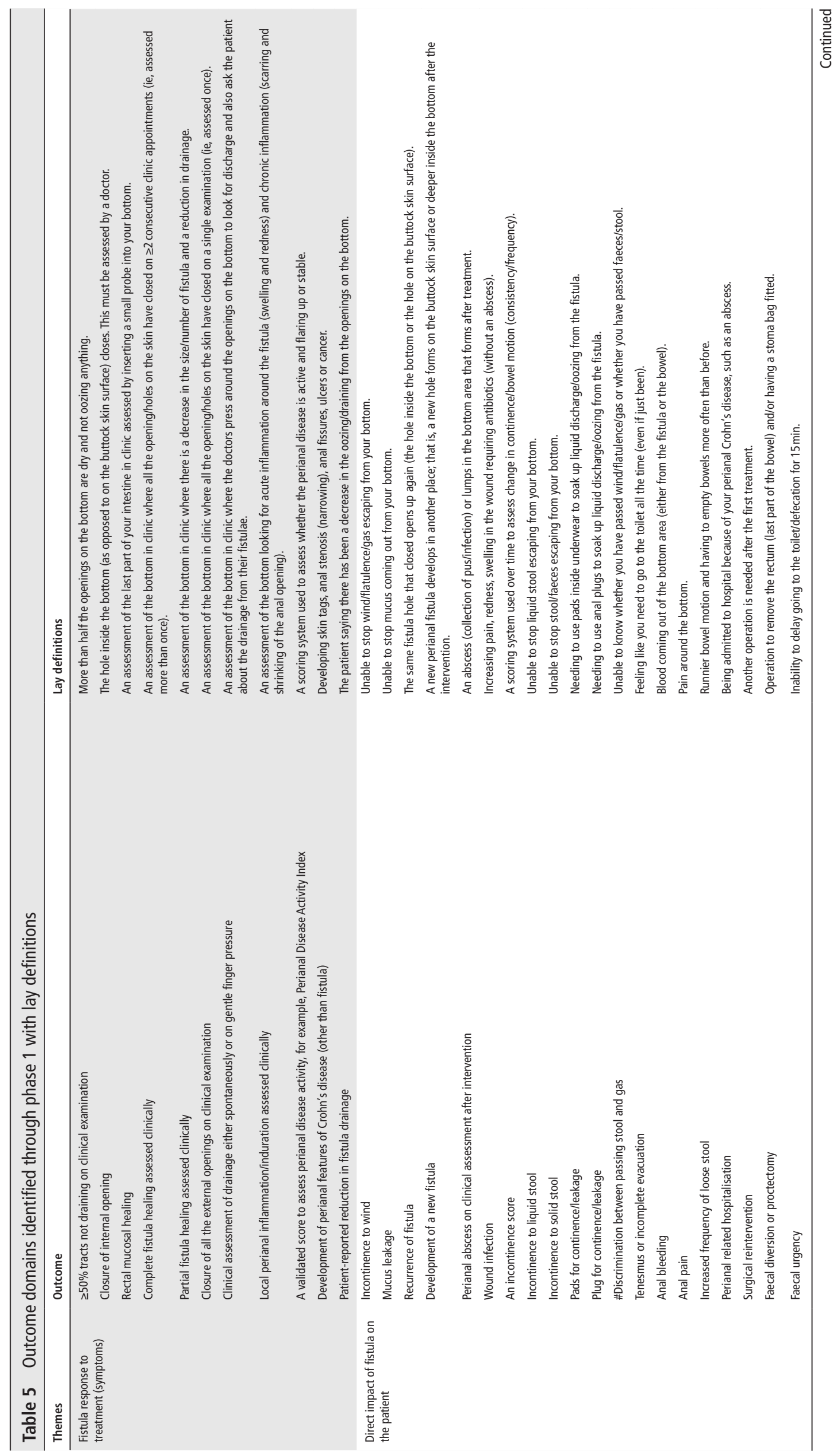

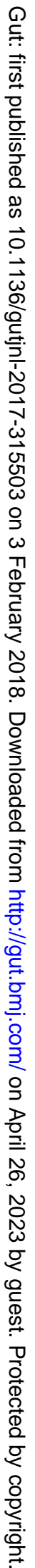




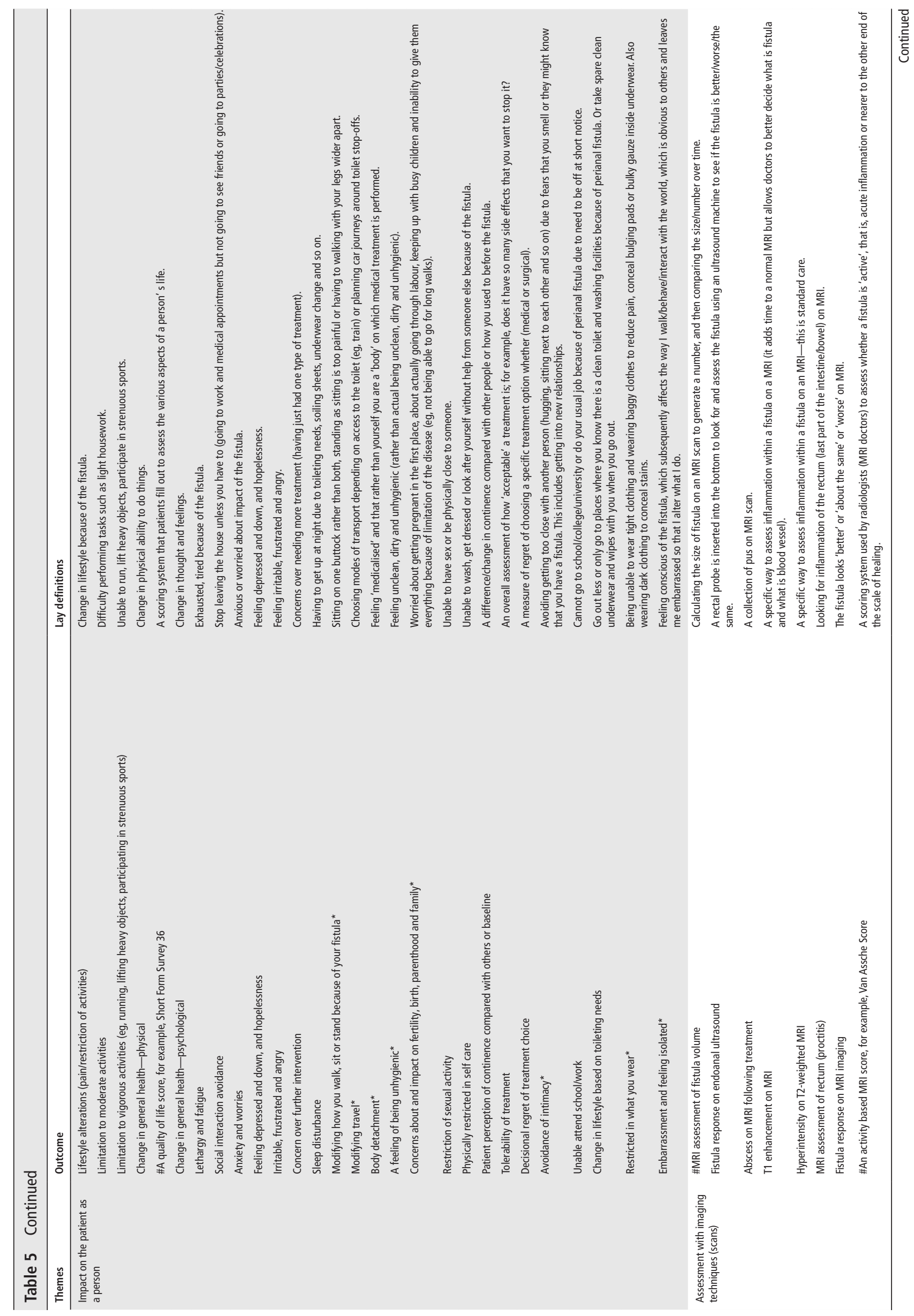

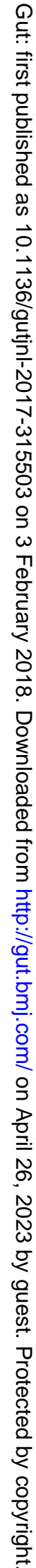




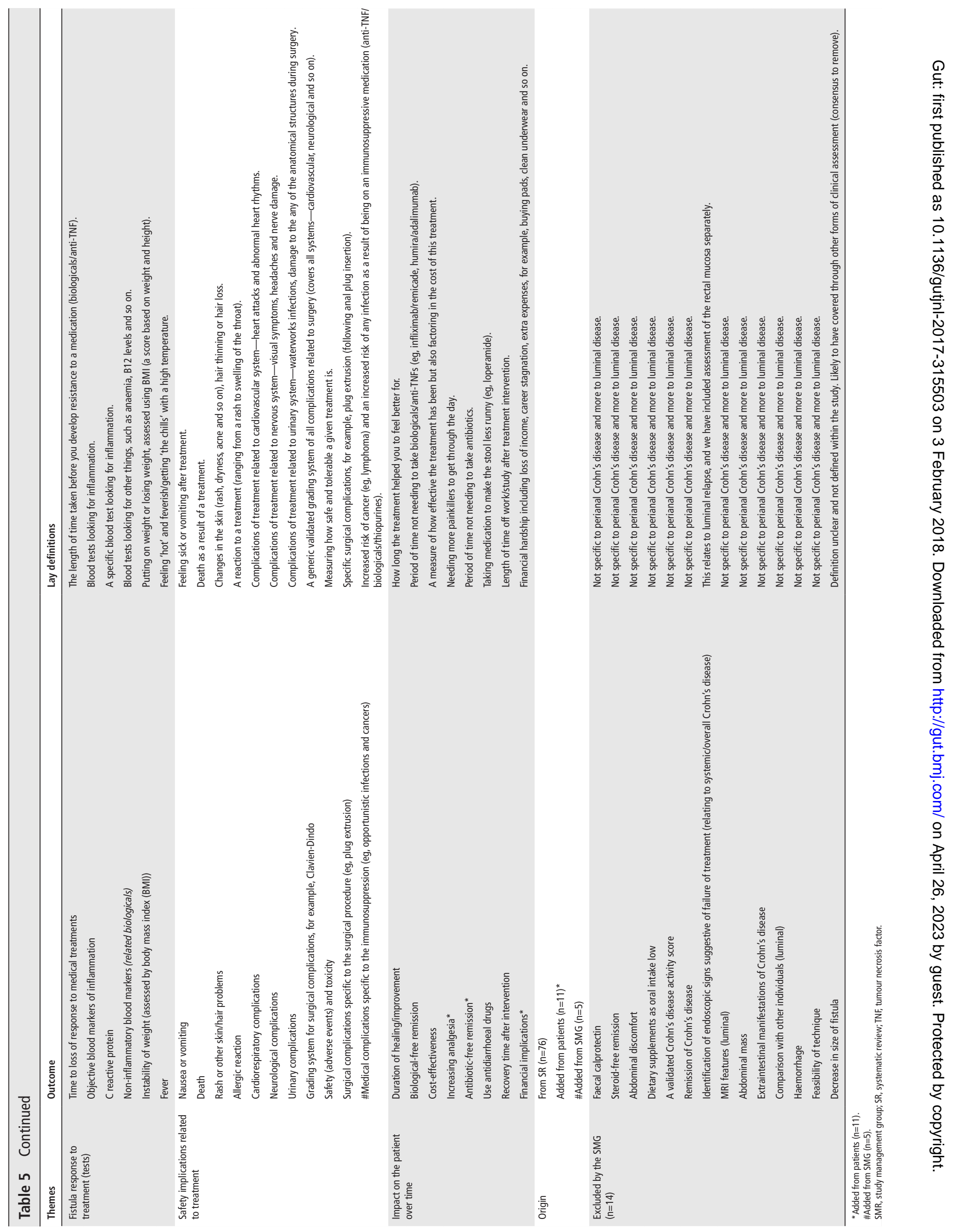


Table 6 Top 10 outcomes voted after phase 1

\begin{tabular}{|c|c|c|c|}
\hline Item & Outcomes & $\begin{array}{l}\text { Panel SuRa } \\
\text { high }\end{array}$ & $\begin{array}{l}\text { Panel SuRa } \\
\text { low }\end{array}$ \\
\hline 30 & Faecal diversion or proctectomy & 97 & 0 \\
\hline 75 & Death & 97 & 3 \\
\hline 15 & Development of a new fistula & 89 & 0 \\
\hline 20 & Incontinence to solid stool & 89 & 0 \\
\hline 28 & Perianal-related hospitalisation & 89 & 0 \\
\hline 29 & Surgical reintervention & 89 & 2 \\
\hline 14 & Recurrence of fistula & 86 & 0 \\
\hline 16 & $\begin{array}{l}\text { Perianal abscess on clinical assessment after } \\
\text { intervention }\end{array}$ & 86 & 2 \\
\hline 56 & Unable attend school/work & 84 & 0 \\
\hline \multirow[t]{2}{*}{19} & Incontinence to liquid stool & 83 & 0 \\
\hline & & $\begin{array}{l}\text { Panel GaNu } \\
\text { high }\end{array}$ & $\begin{array}{l}\text { Panel GaNu } \\
\text { low }\end{array}$ \\
\hline 75 & Death & 100 & 0 \\
\hline 30 & Faecal diversion or proctectomy & 98 & 0 \\
\hline 15 & Development of a new fistula & 97 & 0 \\
\hline 84 & $\begin{array}{l}\text { Medical complications specific to the } \\
\text { immunosuppression (eg, opportunistic } \\
\text { infections and cancers) }\end{array}$ & 97 & 0 \\
\hline 14 & Recurrence of fistula & 94 & 0 \\
\hline 20 & Incontinence to solid stool & 94 & 0 \\
\hline 28 & Perianal-related hospitalisation & 94 & 0 \\
\hline 19 & Incontinence to liquid stool & 92 & 0 \\
\hline 56 & Unable attend school/work & 92 & 0 \\
\hline \multirow[t]{2}{*}{21} & Pads for continence/leakage & 91 & 0 \\
\hline & & $\begin{array}{l}\text { Panel Pa } \\
\text { high }\end{array}$ & $\begin{array}{l}\text { Panel Pa } \\
\text { low }\end{array}$ \\
\hline 19 & Incontinence to liquid stool & 96 & 0 \\
\hline 30 & Faecal diversion or proctectomy & 96 & 0 \\
\hline 20 & Incontinence to solid stool & 93 & 1 \\
\hline 16 & $\begin{array}{l}\text { Perianal abscess on clinical assessment after } \\
\text { intervention }\end{array}$ & 92 & 6 \\
\hline 15 & Development of a new fistula & 92 & 0 \\
\hline 28 & Perianal-related hospitalisation & 91 & 3 \\
\hline 75 & Death & 91 & 5 \\
\hline 17 & Wound infection & 90 & 0 \\
\hline 39 & Social interaction avoidance & 89 & 6 \\
\hline 29 & Surgical reintervention & 86 & 5 \\
\hline
\end{tabular}

Panel GaNu, gastroenterologists and IBD specialist nurses; panel Pa, patients; panel SuRa, colorectal surgeons and radiologists.

\section{DISCUSSION}

This is the first study to standardise outcome reporting in fistulising pCD. An exhaustive list of candidate outcome measures was generated through a systematic review of contemporary outcomes, together with patient views. Using an online tool, a large number of stakeholders were able to participate in a pragmatic Delphi consensus process that ensured emphasis on patient perspective and clinical relevance. Consensus voting and discussion generated a COS that all stakeholders supported with applicability to all study designs. The aim is to improve research and to use this COS as an impetus to drive improvement in clinical management within the field. The COS allows measurement of outcomes for all cohorts of adult patients with pCD, regardless of disease state, the intervention under assessment or the presence of luminal disease. In this study, we established an eight-domain COS for use in studies evaluating interventions in fistulising pCD. A two-domain radiological module is also strongly advocated for use wherever possible.

Fistulising pCD denotes a severe and disabling disease course characterised by the need for multiple hospitalisations and operations. It also has a high economic cost, particularly with the use of biological agents as the mainstay of treatment. ${ }^{23-26}$ The James Lind Alliance, a national Priority Setting Partnership group of patients and clinicians, identified pCD and specifically the individual factors that influence various treatment strategies and outcomes as one of the 'Top 10 unanswered questions' in the field of IBD. ${ }^{27}$ However, the criteria by which success is measured in the management of Crohn's perianal fistula have hitherto been a challenge to researchers. ${ }^{28}$ Most studies have used a measure of the degree of clinical healing as their primary outcome; however, deep tissue healing has been shown to lag behind simple closure of the external openings ${ }^{29}$ and, crucially, success measured this way has generally been disappointingly poor. Moreover, if control of symptoms is the primary objective of treatment, these measures are wholly inappropriate and will fail to demonstrate a benefit, even if patients perceive one. The multimodal treatment of perianal fistulas in Crohn's disease: seton versus antiTNF $\alpha$ versus advancement plasty (PISA) trial group have proposed a set of primary outcomes, which aim to address this deficit. ${ }^{30}$ Of note, the primary outcome is pragmatic and clinically relevant, measuring reinterventions following treatment. The secondary endpoints include quality of life, disease activity and importantly use an MRI-based assessment to determine fistula closure as a long-term measure at 18 months. Recently, the first randomised controlled trial of stem cell therapy in fistulising pCD was published. ${ }^{31}$ The authors used a new composite primary endpoint, comprising clinical and radiological healing, further revealing the lack of agreement on which fistula outcomes to use and also recognising the inadequacy of a single outcome measure to define success.

The International Consortium for Health Outcome Measurement (ICHOM) has recently been published for IBC. It emphasises the importance of patient-reported outcome measures in IBD research but as it has been designed for IBD in general it has minimal information for patients with fistulas. ${ }^{32}$ This COS aims to address an unmet need in pCD where standardising outcome measurement is particularly difficult due to changes in the patient's goals of treatment over time, the refractory nature of perianal disease compared with luminal disease and the specific symptoms associated with the condition. ${ }^{33} 34$

A strength of this study is that every stage of the process, including amalgamation, addition and exclusion of outcomes, was performed by consensus and always included patient representation. Examples include the interventional complications and morbidity, which featured in the outcomes presented on the consensus day but were excluded from the final COS, as the participants felt these would be reported as a minimum in the Preferred Reporting Items for Systematic Reviews and Meta-Analyses harms checklist. ${ }^{35}$ A similar discussion excluded 'death' as an outcome. The initial design of this study followed the guide set by the OMERACT ${ }^{36}$ group, but at the consensus meeting, it was felt that 'death' was less suitable for this COS and a more relevant hard endpoint would be 'faecal diversion'. Another consensus decision was that of an optional imaging module, but because MRI is not universally accessible, it did not reach threshold for inclusion as a mandatory outcome measure. Another strength was the diversity in the study management group with stakeholder leads and methodologists from a number of different institutions and geographical locations, and the crucial stakeholders (patients) were always prioritised. For example, we found that patients tended to rank most outcomes highly, gastroenterologists slightly less so and surgeons least of 
Table 7 Outcomes to be included in the $\operatorname{COS}$ at the consensus meeting

\begin{tabular}{|c|c|c|c|c|c|c|c|}
\hline & \multirow[b]{2}{*}{ Question } & \multicolumn{3}{|l|}{$\% \ln$} & \multirow[b]{2}{*}{ Overall (\%) } & \multirow{2}{*}{$\begin{array}{l}\text { Patient vote } \\
\text { alone }(\%)\end{array}$} & \multirow{2}{*}{$\begin{array}{l}\text { Final } \\
\text { consensus }\end{array}$} \\
\hline & & Panel SuRa (\%) & Panel GaNu (\%) & Panel Pa (\%) & & & \\
\hline 1 & Patient-reported reduction in fistula drainage & 57.1 & 53.3 & 30.0 & 46.8 & & \\
\hline 2 & Development of other perianal features & 53.8 & 73.3 & 70.0 & 65.7 & & \\
\hline 3 & Complete fistula healing assessed clinically & 53.3 & 40.0 & 44.4 & 45.9 & & \\
\hline 4 & Validated score to assess perianal disease activity & 100.0 & 93.8 & 77.8 & 90.5 & & IN \\
\hline 5 & Development of a new fistula & 69.2 & 83.3 & 87.5 & 80.0 & & IN \\
\hline 6 & Incontinence to solid stool & 26.7 & 33.3 & 40.0 & 33.3 & & \\
\hline 7 & Pads for continence/leakage & 26.7 & 40.0 & 40.0 & 35.6 & & \\
\hline 8 & Recurrence of fistula & 85.7 & 68.8 & 80.0 & 78.2 & & \\
\hline 9 & Perianal abscess on clinical assessment after intervention & 86.7 & 100.0 & 90.0 & 92.2 & & IN \\
\hline 10 & Incontinence to liquid stool & 71.4 & 93.3 & 100.0 & 88.3 & & Combinedt \\
\hline 11 & Perianal-related hospitalisation & 35.7 & 50.0 & 10.0 & 31.9 & & \\
\hline 12 & Faecal diversion or proctectomy & 100.0 & 100.0 & 100.0 & 100.0 & & IN \\
\hline 13 & Wound infection & 0.0 & 0.0 & 30.0 & 10.0 & & \\
\hline 14 & Anal pain & 66.7 & 87.5 & 90.0 & 81.4 & & \\
\hline 15 & Discrimination between passing stool and gas & 6.7 & 33.3 & 12.5 & 17.5 & & \\
\hline 16 & Surgical reintervention & 93.3 & 93.8 & 80.0 & 89.0 & & IN \\
\hline 17 & Anal bleeding & 0.0 & 14.3 & 60.0 & 24.8 & & \\
\hline 18 & Reversal of defunctioning stoma & 33.3 & 37.5 & 22.2 & 31.0 & & \\
\hline 19 & A global assessment of incontinence that covers all aspects of leakage & 92.9 & 100.0 & 100.0 & 97.6 & & IN \\
\hline 20 & Fistula response on MRI & 85.7 & 86.7 & 80.0 & 84.1 & & IN \\
\hline 21 & Hyperintensity on T2-weighted MRI & 46.7 & 38.5 & 62.5 & 49.2 & & \\
\hline 22 & MRI assessment of fistula volume & 35.7 & 53.8 & 55.6 & 48.4 & & \\
\hline 23 & Fistula T1 enhancement on MRI & 13.3 & 45.5 & 60.0 & 39.6 & & \\
\hline 24 & Abscess on MRI following treatment & 66.7 & 87.5 & 60.0 & 71.4 & & \\
\hline 25 & An activity-based MRI score & 86.7 & 93.3 & 88.9 & 89.6 & & IN \\
\hline 26 & A global quality of life score & 100.0 & 93.8 & 100.0 & 97.9 & $\mathrm{n} / \mathrm{a}$ & IN \\
\hline 27 & Physically restricted in caring for oneself & 15.4 & 26.7 & 70.0 & 37.4 & 70.0 & \\
\hline 28 & Change in lifestyle based on toileting needs & 23.1 & 53.3 & 87.5 & 54.6 & 90.0 & IN \\
\hline 29 & Embarrassment and feeling bloated & 25.0 & 42.9 & 60.0 & 42.6 & 60.0 & \\
\hline 30 & Unable to attend school/work & 36.4 & 86.7 & 100.0 & 74.3 & 100.0 & IN \\
\hline 31 & Restriction of sexual activity & 58.3 & 80.0 & 90.0 & 76.1 & 100.0 & IN \\
\hline 32 & Lethargy and fatigue & 0.0 & 20.0 & 66.7 & 28.9 & 60.0 & \\
\hline 33 & Limitation to moderate activities & 8.3 & 12.5 & 30.0 & 16.9 & 40.0 & \\
\hline 34 & Change in general health & 16.7 & 64.3 & 80.0 & 53.7 & 80.0 & \\
\hline 35 & Avoidance of intimacy & 36.4 & 60.0 & 88.9 & 61.8 & 88.9 & Combined $\ddagger$ \\
\hline 36 & Anxiety and worries & 8.3 & 25.0 & 55.6 & 29.6 & 75.0 & \\
\hline 37 & Change in physical ability to do things & 0.0 & 12.5 & 77.8 & 30.1 & 80 & \\
\hline 38 & Feeling depressed, down, hopeless, unable to cope & 7.7 & 42.9 & 90.0 & 46.8 & 90 & IN \\
\hline 39 & Modifying how you sit, walk and stand because of your fistula & 33.3 & 57.1 & 70.0 & 53.5 & 80 & \\
\hline 40 & Lifestyle alterations (pain/restriction) & 25.0 & 53.8 & 80.0 & 52.9 & 100 & IN \\
\hline 41 & Social interaction avoidance & 50.0 & 46.2 & 70.0 & 55.4 & 80 & \\
\hline 42 & Duration of improvement & 53.8 & 80.0 & 88.9 & 74.2 & & \\
\hline 43 & Death & 46.2 & 42.9 & 55.6 & 48.2 & & \\
\hline 44 & Allergic reaction & 7.7 & 6.7 & 37.5 & 17.3 & & \\
\hline 45 & Safety (adverse events) and toxicity & 7.7 & 7.1 & 50.0 & 21.6 & & \\
\hline 46 & Urinary complications & 38.5 & 76.9 & 77.8 & 64.4 & & \\
\hline 47 & Cardiorespiratory complications & 7.7 & 0.0 & 44.4 & 17.4 & & \\
\hline 48 & Neurological complications & 0.0 & 8.3 & 60.0 & 22.8 & & \\
\hline 49 & Medical complications specific to immunosuppression & 0.0 & 15.4 & 44.4 & 19.9 & & \\
\hline
\end{tabular}

*'Patient Priorities' - consensus agreement by all to allow these items to be voted on by patient alone.

†Combined with 'A global assessment of incontinence that covers all aspects of leakage'.

$\ddagger$ Combined with 'Restriction of sexual activity'.

COS, core outcome set.

all. This meant that the outcomes that fell below the inclusion bar tended to do so as a result of the views of the clinicians, rather than the patients, potentially deviating from the aim of a patient-centred COS. There is currently no guidance on how to discuss outcomes with patients and carers in qualitative research, ${ }^{37}$ but in efforts to ameliorate this effect, the study management group used separate thresholds for clinicians and patients to allow for prioritisation of outcomes scored particularly highly by the patients. In addition, some outcomes seemed to score poorly despite very similar outcomes scoring highly. 
PHASE 1 - DOMAIN GENERATION

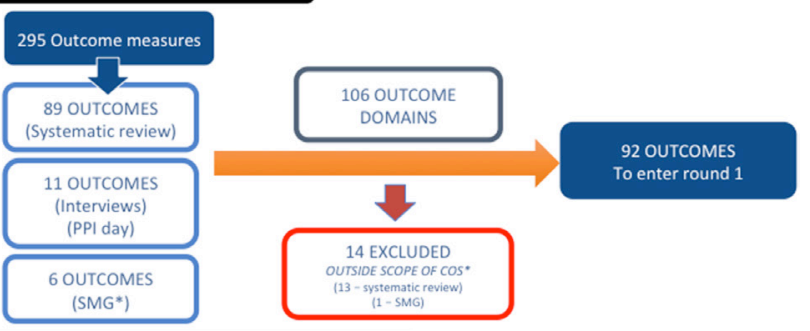

PHASE 2 - DELPHI PROCESS

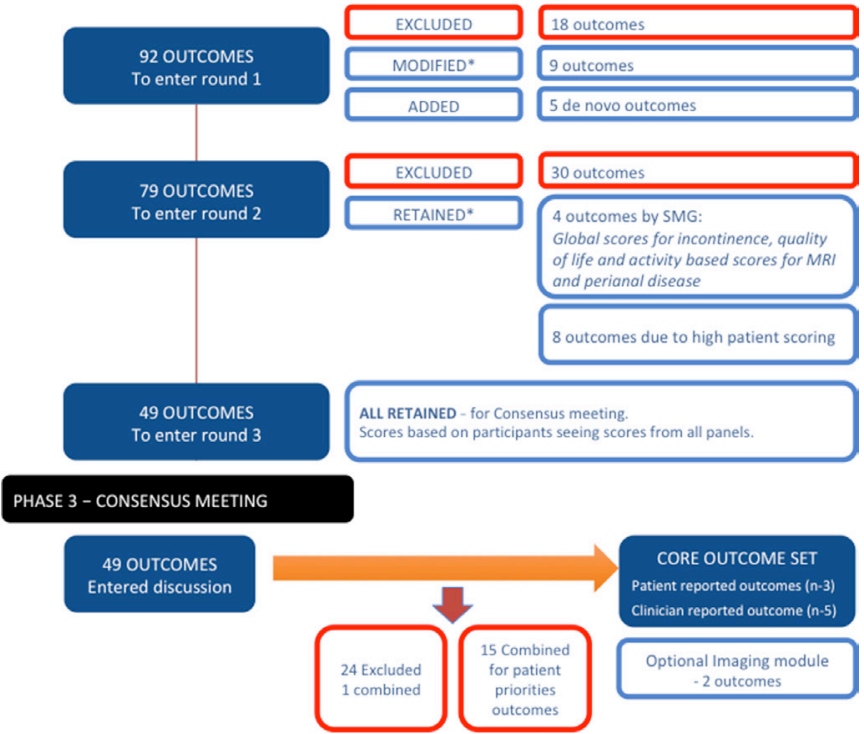

Figure 2 Outcome flow diagram. COS, core outcome set; PPI, patient and public involvement.

This was taken as evidence of poorly worded descriptions or concepts difficult to explain in pithy prose. For example, the concept of a global assessment of quality of life reached the threshold for exclusion in the online Delphi but was discussed

Box 2 Core outcome set for fistulising perianal Crohn's disease

Patient-reported outcomes

- Global assessment of quality of life.

- Combined score of patient priorities.

- Lifestyle restriction (general)

- Lifestyle restriction based on toileting needs

- Depression

- Inability to attend school/work

- Restriction of sexual activity and avoidance of intimacy.

- Global assessment of incontinence.

Clinician-reported outcomes

- A validated score to assess perianal disease activity.

- Development of a perianal abscess.

- Development of a new/recurrent fistula.

- Unplanned surgical reintervention.

- Faecal diversion or proctectomy.

Imaging (optional module)

- Fistula response on MRI.

- An activity-based MRI score responsive to change. at the consensus meeting for the reasons above and was selected as part of the final COS once it was adequately explained to the patients by whom it was then championed.

A limitation of this study is that all the participants were based in the UK, which could potentially affect generalisability to other populations, especially in low-income countries. There was an active decision by the study management group to minimise attrition through the rounds and to achieve maximum recruitment. One missing stakeholder was industry; this was discussed and specifically excluded due to concern about potential bias. An online survey was chosen due to its ease of use, increasing the feasibility of national sampling, as well as removing interview bias. ${ }^{38}$ To date, this study has one of the largest numbers of participants and one of the lowest attrition rates.

Given the poor rates of fistula closure experienced by most patients, the range of new medical and surgical treatments emerging and the high cost of many of them determining their relative efficacy and cost effectiveness is important. Comparison of different techniques from different studies mandates standardised outcome reporting, which this COS will provide. Outcomes specific to a given study as a result of the technique used or population studied, such as the rate of plug extrusion, or complications relating to immunosuppression, remain important to measure and are not excluded by a COS. Innovation, progress and pragmatism will require researchers to measure other outcomes, but in order to appraise interventions completely and ensure relevance to patients, a COS should also be used. Although MRI is crucial to determine deep tissue healing, its value in the assessment of symptom palliation is less clear, and it is not readily available to all studies or institutions, so it could not be considered a core outcome but is a strongly advocated addition where appropriate.

The Evaluating goal-directed management of fistulising perianal Crohn's disease research group is building a portfolio of foundation research, of which this COS is a central part. This COS requires international validation if it is to be used outside the UK. It is also necessary to develop a core measurement set, a collection of measurement tools and standards by which these outcomes can be assessed in a given study. For example, there is no validated, disease-specific, patient-derived quality of life measurement tool for Crohn's anal fistula. This is another 'unmet need' described by the European Crohn's and Colitis Organisation's consensus group. ${ }^{20}$ Creation of such a measurement tool is underway, and development of a core measurement set to complement this COS is also underway.

\section{CONCLUSION}

Using rigorous methodology and representative stakeholder engagement, we have generated a COS for use in fistulising pCD studies. Groups assessing treatment in fistulising pCD should be strongly encouraged to adopt and use this COS to reduce the heterogeneity of outcome reporting and improve the quality and comparability of future research.

Twitter@KSahnan@StMarksHospital @COMETinitative @StMarksFRU @ philtozer1@AzminaVerjee@AngusGKMcNair@DanHind1 @wannabehaweye @ stevebrownsheff @DrAilsaHart @ACPGBI @BritSocGasto @BDRF1 \#CRStrials

Collaborators Abercrombie John; Acheson Austin; Anderson Simon; Armstrong Aidan; Arthur James; Ashton Katherine; Babu Sathish; Beales lan; Bhatnagar Gauraang; Bloom Stuart; Blunt Dominic; Brown Matthew; Burling David; Butterworth Jeffrey; Calvert Christopher; Cooney Rachel; Coyne Peter; Creed Thomas; Cuming Tamzin; Dennis Robert; Douie Walter; Dunkley Irene; Eaden Jayne; Epstein Jonathan; Evans Martyn; Fallis Simon; Fieldsend Beverley; Fraser Catherine; Goodhand James; Grierson Catherine; Griffiths Ben; Gupta Arun; Gupta Sanjay; Guy Richard; Hall Alex; Hall Diane; Hall Nigel; Halligan Steve; Hancock Laura; Harbord Marcus; Hargest Rachel; Hawthorne Barney; Hayee Bu; Helbren Emma; Henderson Paul; Hoare Tim; Holtham Stephen; Ilangovan Rajapandian; Johnson Matthew; Kemp Cheryl; Nicholas 
Kennedy A; Kiparissi Fevronia; Knowles Charles; Lee Bee; Lewis Wendy; Limdi Jimmy; Lovegrove Richard; MacLean Peter; Maginnis Janis; Mann Steven; Mansfield John; Marshall Michele; Maxwell-Armstrong Charles; McNair Alistair; Nahal Jasbir; Pallan Arvind; Parkes Gareth; Patel Rajan; Patel Uday; Pee Leon; Phillis Kathryn; Pinkney Thomas; Planche Katie; Pollok Richard; Power Niall; Puckett Mark; Razack Abdul; Robinson Kerry; Rogers Dan; Richard Russell K; Rutter Mathew; Ryan Suzanne; Salaman Judith; Saunders John; Selinger Christian; Shaikh Irshad; Shaw lan; Singh Baljit; Sitchon Ederis; Smart Christopher; Smart Neil; Stearns Adam; Stubbs Ben; Stuart Taylor A; Teare Julian; Tony Tham C; Thomas Pradeep; Todd John; Torkington Jared; Travis Simon; Tremelling Mark; Tyrrell Tracey; Tzivinikos Christos; Vaizey Carolynne; Warren Oliver; Warusavitarne Janindra; Wesley Emma; West Debbie; Whitehead Emma; Williams Graham; Wills Mark; Wilson Graeme; Wood Eleanor; Yarrow Hannah; Younge Lisa

Contributors All authors were involved in the design and delivery of the project. The manuscript was written by KS, PJT, SOA, AGKM, ALH and NSF. All authors reviewed and critically appraised the manuscript before submission.

Funding KS is supported by a Royal College of Surgeons of England Research Scholarship. AGKM is supported by the NIHR Biomedical Research Centre at the University Hospitals Bristol NHS Foundation Trust and the University of Bristol. The study was supported by the Bowel Disease Research Foundation (BDRF), the Royal College of Surgeons, of England (RCS) and the Association of Coloproctologists of Great Britain and Ireland (ACPGBI)

Disclaimer The views expressed are those of the authors and not necessarily those of the UK National Health Service, National Institute for Health Research or Department of Health.

Competing interests KS and SOA have received honoraria from Takeda for sitting on an advisory board. PJT has received honoraria from Takeda for sitting on an advisory board and for speaking at a symposium. AJL is an advisory board member or received lecture fees from Takeda Pharma, AbbVie, Vifor Pharma, Dr Falk and Shield Therapeutics. ALH has served as a consultant, advisory board member or speaker for AbbVie, Atlantic, Bristol-Myers Squibb, Celltrion, Falk, Ferring, Janssen, MSD, Napp Pharmaceuticals, Pfizer, Pharmacosmos, Shire and Takeda. She also serves on the Global Steering Committee for Genentech. NSF has received travel grants from Obsidian Health and Strategen to deliver invited lectures.

Provenance and peer review Not commissioned; externally peer reviewed.

Data sharing statement Additional supplementary data are available online. All other data are available on request.

Open access This is an open access article distributed in accordance with the Creative Commons Attribution Non Commercial (CC BY-NC 4.0) license, which permits others to distribute, remix, adapt, build upon this work non-commercially, and license their derivative works on different terms, provided the original work is properly cited and the use is non-commercial. See: http://creativecommons.org/ licenses/by-nc/4.0/

C) Article author(s) (or their employer(s) unless otherwise stated in the text of the article) 2019. All rights reserved. No commercial use is permitted unless otherwise expressly granted.

\section{REFERENCES}

1 Silverberg MS, Satsangi J, Ahmad T, et al. Toward an integrated clinical, molecular and serological classification of inflammatory bowel disease: report of a Working Party of the 2005 Montreal World Congress of Gastroenterology. Can I Gastroenterol 2005;19 Suppl A:5A-36.

2 Afsarlar CE, Karaman A, Tanır G, et al. Perianal abscess and fistula-in-ano in children: clinical characteristic, management and outcome. Pediatr Surg Int 2011;27:1063-8.

3 Sahnan K, Adegbola SO, Tozer PJ, et al. Perianal abscess. BMJ 2017;356:1475.

4 Tozer PJ, Burling D, Gupta A, et al. Review article: medical, surgical and radiological management of perianal Crohn's fistulas. Aliment Pharmacol Ther 2011;33:5-22.

5 Panés J, García-Olmo D, Van Assche G, et al. Expanded allogeneic adipose-derived mesenchymal stem cells (Cx601) for complex perianal fistulas in Crohn's disease: a phase 3 randomised, double-blind controlled trial. The Lancet 2016;388:1281-90.

6 Meinero P, Mori L. Video-assisted anal fistula treatment (VAAFT): a novel sphincter-saving procedure for treating complex anal fistulas. Tech Coloproctol 2011;15:417-22.

7 Wilhelm A. A new technique for sphincter-preserving anal fistula repair using a novel radial emitting laser probe. Tech Coloproctol 2011;15:445-9.

8 Garcia-Olmo D, Herreros D, Pascual I, et al. Expanded adipose-derived stem cells for the treatment of complex perianal fistula: a phase II clinical trial. Dis Colon Rectum 2009:52:79-86.

9 Ellis CN. Outcomes with the use of bioprosthetic grafts to reinforce the ligation of the intersphincteric fistula tract (BioLIFT procedure) for the management of complex anal fistulas. Dis Colon Rectum 2010:53:1361-4.
10 Han JG, Wang ZJ, Zheng Y, et al. Ligation of intersphincteric fistula tract vs Ligation of the intersphincteric fistula tract plus a bioprosthetic anal fistula plug procedure in patients with transsphincteric anal fistula: early results of a multicenter prospective randomized trial. Ann Surg 2015.

11 Allin B, Knight M, Allin B. Trials NETS1G study: development of a core outcome set for use in determining the overall success of gastroschisis treatment. 2015.

12 Gargon E, Gurung B, Medley N, et al. Choosing important health outcomes for comparative effectiveness research: a systematic review. PLoS One 2014;9:e99111.

13 Gargon E, Williamson PR, Altman DG, et al. The COMET Initiative database: progress and activities update (2015). Trials 2017;18:54

14 Klokker L, Tugwell P, Furst DE, et al. Developing an omeract core outcome set for assessing safety components in rheumatology trials: the OMERACT safety working group. J Rheumatol 2017;44.

15 Allin B, Bradnock T, Kenny S, et al. NETS1HD: study protocol for development of a core outcome set for use in determining the overall success of Hirschsprung's disease treatment. Trials 2016;17:577

16 McNair AG, Whistance RN, Forsythe RO, et al. Core outcomes for colorectal cancer surgery: a consensus study. PLoS Med 2016;13:e1002071-14.

17 Kirkham JJ, Clarke M, Williamson PR. A methodological approach for assessing the uptake of core outcome sets using ClinicalTrials.gov: findings from a review of randomised controlled trials of rheumatoid arthritis. BMJ 2017;357:j2262.

18 Williamson PR, Altman DG, Blazeby JM, et al. Developing core outcome sets for clinical trials: issues to consider. Trials 2012;13:132.

19 Kirkham JJ, Gorst S, Altman DG, et al. Core outcome set-standards for reporting: the COS-STAR statement. PLoS Med 2016;13:e1002148.

20 Gecse KB, Bemelman W, Kamm MA, et al. A global consensus on the classification, diagnosis and multidisciplinary treatment of perianal fistulising Crohn's disease. Gut 2014:63:1381-92

21 Nuha A, Robin K, Ailsa L, et al. The anal fistula journal: effects on patients' life experiences. In: Gastroenterology. 2014;146:S-207--0.

22 Harman NL, Bruce IA, Callery P, et al. MOMENT-Management of Otitis Media with Effusion in Cleft Palate: protocol for a systematic review of the literature and identification of a core outcome set using a Delphi survey. Trials 2013;14:70.

23 Chaparro M, Zanotti C, Burgueño P, et al. Health care costs of complex perianal fistula in Crohn's disease. Dig Dis Sci 2013;58:3400-6.

24 Juan J, Estiarte R, Colomé E, et al. Burden of illness of Crohn's disease in Spain. Dig Liver Dis 2003;35:853-61 http://www.ncbi.nlm.nih.gov/pubmed/14703880.

25 Silverstein MD, Loftus EV, Sandborn WJ, et al. Clinical course and costs of care for Crohn's disease: Markov model analysis of a population-based cohort. Gastroenterology 1999;117:49-57 http://www.ncbi.nlm.nih.gov/pubmed/ 10381909.

26 Baji P, Gulácsi L, Brodszky V, et al. Cost-effectiveness of biological treatment sequences for fistulising Crohn's disease across Europe. United Eur Gastroenterol J 2017:1-12.

27 James Lind Alliance. Inflammatory Bowel Disease Top 10. 2015 http://www.jla.nihr. ac.uk/priority-setting-partnerships/inflammatory-bowel-disease/top-10-priorities/ (accessed 14 Jun 2017).

28 Tiernan J, Cook A, Geh I, et al. Use of a modified Delphi approach to develop research priorities for the Association of Coloproctology of Great Britain and Ireland. Colorectal Disease 2014;16:965-70.

29 Tozer P, Ng SC, Siddiqui MR, et al. Long-term MRI-guided combined anti-TNF- $\alpha$ and thiopurine therapy for Crohn's perianal fistulas. Inflamm Bowel Dis 2012;18:1825-34.

30 de Groof EJ, Buskens CJ, Ponsioen CY, et al. Multimodal treatment of perianal fistulas in Crohn's disease: seton versus anti-TNF versus advancement plasty (PISA): study protocol for a randomized controlled trial. Trials 2015;16:366.

31 Panés J, García-Olmo D, Van Assche G, et al. Expanded allogeneic adipose-derived mesenchymal stem cells (Cx601) for complex perianal fistulas in Crohn's disease: a phase 3 randomised, double-blind controlled trial. Lancet 2016:388:1281-90.

32 Kim AH, Roberts C, Feagan BG, et al. Developing a standard set of patient-centred outcomes for inflammatory bowel disease - an International, cross-disciplinary consensus. J Crohns Colitis 2017.

33 Tarrant KM, Barclay ML, Frampton CM, et al. Perianal disease predicts changes in Crohn's disease phenotype-results of a population-based study of inflammatory bowel disease phenotype. Am I Gastroenterol 2008;103:3082-93.

34 Beaugerie L, Seksik P, Nion-Larmurier I, et al. Predictors of Crohn's disease. Gastroenterology 2006;130:650-6.

35 Zorzela L, Loke YK, loannidis JP, et al. PRISMA harms checklist: improving harms reporting in systematic reviews. BMJ 2016;i:i157.

36 Boers M, Kirwan JR, Wells G, et al. Developing core outcome measurement sets for clinical trials: OMERACT filter 2.0. J Clin Epidemiol 2014;67:745-53.

37 Jones JE, Jones LL, Keeley TJ, et al. A review of patient and carer participation and the use of qualitative research in the development of core outcome sets. PLoS One 2017:12:e0172937.

38 Van Selm M, Jankowski NW. Conducting online surveys. Quality and Quantity 2006:40:435-56. 